\title{
Effect Handlers for the Masses
}

\author{
JONATHAN IMMANUEL BRACHTHÄUSER, PHILIPP SCHUSTER, and KLAUS OSTER- \\ MANN, University of Tübingen, Germany
}

Effect handlers are a program structuring paradigm with rising popularity in the functional programming language community and can express many advanced control flow abstractions. We present the first implementation of effect handlers for Java - an imperative, object oriented programming language. Our framework consists of three core components: A type selective CPS transformation via JVM bytecode transformation, an implementation of delimited continuations on top of the bytecode transformation and finally a library for effect handlers in terms of delimited continuations.

CCS Concepts: • Software and its engineering $\rightarrow$ Control structures; Source code generation; Runtime environments; Abstraction, modeling and modularity;

Additional Key Words and Phrases: effect handlers, algebraic effects, delimited continuations, java, jvm, bytecode transformation

\section{ACM Reference Format:}

Jonathan Immanuel Brachthäuser, Philipp Schuster, and Klaus Ostermann. 2018. Effect Handlers for the Masses. Proc. ACM Program. Lang. 2, OOPSLA, Article 111 (November 2018), 27 pages. https://doi.org/10.1145/3276481

\section{INTRODUCTION}

Algebraic effects [Plotkin and Power 2003] in their extension with effect handlers [Plotkin and Pretnar 2009] are a program structuring paradigm, splitting programs into three parts: (1) effect signatures, that declare effect operations like for example yield to output an element of a (push-based) stream, getHttp to send an (potentially asynchronous) http-request, raise to throw an exception and so on, (2) effectful functions, that call these effect operations either directly or indirectly via other effectful functions, (3) effect handlers [Bauer and Pretnar 2013; Plotkin and Pretnar 2009], that implement the effect operations, specifying what it means for example to yield, send http-requests or throw exceptions.

Lacking a general mechanism, many control flow abstractions in Java like generators ${ }^{1}$, asynchronous programming with async/await ${ }^{2}$, the coroutine programming model $^{3}$ and fibers (lightweight user-level threads) ${ }^{4}$ are currently implemented by custom source-to-source or bytecode transformations. Since each extension makes different assumptions about the generated code, combining the different concepts in a single project is non-trivial. As has been shown in the literature, using effect handlers, many of these control flow abstractions can be expressed as simple libraries [Dolan et al. 2017, 2015; Leijen 2017b], naturally allowing a combined usage. We will revisit some examples of control flow abstractions in the context of Java in Section 4.

\footnotetext{
${ }^{1}$ https://github.com/peichhorn/lombok-pg/wiki/Yield

${ }^{2}$ https://github.com/electronicarts/ea-async

${ }^{3}$ https://github.com/offbynull/coroutines

${ }^{4}$ http://docs.paralleluniverse.co/quasar
}

This work is licensed under a Creative Commons Attribution 4.0 International License.

(C) 2018 Copyright held by the owner/author(s).

2475-1421/2018/11-ART111

https://doi.org/10.1145/3276481

Proc. ACM Program. Lang., Vol. 2, No. OOPSLA, Article 111. Publication date: November 2018. 
Many implementations of effect handlers can be found in functional languages. They are either built into the language, like in Eff [Bauer and Pretnar 2015], Koka [Leijen 2014], Frank [Lindley et al. 2017] and Links [Hillerström et al. 2017], or implemented as a libraries for Haskell [Kammar et al. 2013; Kiselyov et al. 2013; Wu and Schrijvers 2015] or Idris [Brady 2013]. Recently, object-oriented and imperative languages also started to adopt effect handlers, both as a built-in language construct for OCAML [Dolan et al. 2017]), as well as as libraries for OCAML [Kammar et al. 2013; Kiselyov and Sivaramakrishnan 2016], C [Leijen 2017a] and Scala [Brachthäuser and Schuster 2017].

We identify the following important key characteristics of programming languages and libraries with support for effect handlers:

- effectful functions mention the effects they use in their type and an effect type system asserts that all effects are eventually handled;

- effect handlers can be applied locally, describing a dynamic scope in which effect operations of a particular effect signature are handled by this very handler;

- to implement effect operations, handlers gets access to the delimited continuation, that is the remainder of the program up to the enclosing call to the effect handler.

In this paper, we present a framework for programming with effect handlers in Java called EFFEKT ${ }^{5}$. In our framework, effect signatures are Java interfaces, effect handlers are classes that implement those interfaces and effectful functions use instances of the effect handlers. Our implementation consists of three core components: A type selective CPS transformation via JVM bytecode transformation, an implementation of delimited continuations on top of the bytecode transformation and a library for effect handlers in terms of delimited continuations.

While all three components are designed in concert to implement the effect handler library, they can be used and understood individually. The bytecode transformation for example is performed independent of Java as the source language and could be reused with other JVM languages like Scala, Kotlin, JRuby, Clojure and others.

In short, our contributions are:

- The first library design for programming with effect handlers in Java.

- An implementation of multi-prompt delimited continuations in Java. It uses trampolining and avoids the typical linear overhead of restoring the stack upon resumption common to all continuation libraries in Java that we are aware of.

- A type-selective, signature preserving CPS transformation of JVM bytecode. We use closures introduced in Java 1.8 to create specialized instances of continuation frames. The general idea is applicable to any VM-based language that supports closure creation.

- Examples of how to program with effect handlers in Java.

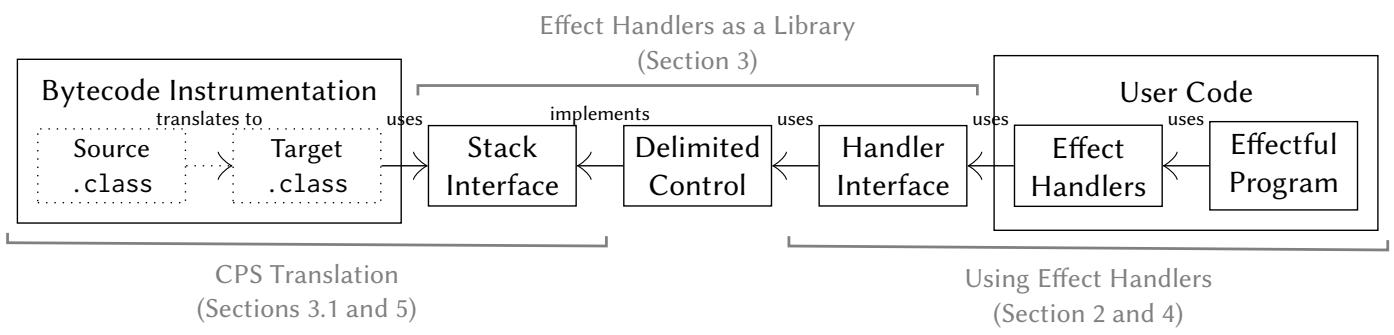

Fig. 1. Structure of the EFfEkT framework. Directed, solid arrows express dependencies.

$\overline{5_{\text {https://github.com/double-blind-review/effekt }}}$ 


\subsection{Overview}

The paper structure relates to the structure of our framework which is presented in Figure 1.

User Code. We show how to program with effect handlers in Java using our library. Section 2 presents the library design of EFFEKT by means of a standard running example of an effectful program; Section 4 illustrates the expressiveness of EFFEKT with several more complex examples.

Bytecode Instrumentation. Section 3.1 illustrates the CPS translation by applying it to the running example and section 5 describes the implementation of the translation in more detail. Section 6.3 evaluates the performance overhead induced by our translation and compares the performance of EFFEKT to other continuation libraries in Java.

Delimited Control. Section 3.2 describes the implementation of multi-prompt delimited continuations [Dyvbig et al. 2007] in Java. It interfaces with the instrumented bytecode by implementing the Stack interface that the instrumented bytecode uses. This means that the bytecode instrumentation component can potentially be used as a backend for applications other than our implementation of delimited continuations and that the instrumentation could be exchanged by another backend that implements the Stack interface.

Effect Handlers as a Library. Subsection 3.3 shows the (to our knowledge) first implementation of effect handlers as a library for Java. We implement the effect handler library on top of multi-prompt delimited continuations as defined in interface DelimitedControl. While the implementation of effect handlers in terms of multi-prompt delimited continuations is not novel [Kiselyov and Sivaramakrishnan 2016], choosing Java as the target language is and poses new challenges like integrating effect handlers with mutable state and designing an API that only uses interfaces and generics for typing. However, it also opens new opportunities like using subtyping and inheritance for handler reuse. The effect handler library is independent of our concrete implementation of DelimitedControl and could for instance be reused together with a modified JVM runtime that directly supports delimited continuations. Section 6.2 discusses design decisions in our effect handler library and compares the performance of EFFEKT with existing effect libraries in Scala.

\section{PROGRAMMING WITH EFFECT HANDLERS IN EFFEKT}

To get a first impression of how to program with effect handlers in EFFEKT, let's look at a standard example from the literature [Kammar et al. 2013] - a drunk tossing a coin (Figure 2). In the effectful function drunkFlip in Subfigure 2a we use the effect operation flip to nondeterministically choose whether the drunk is too drunk to catch the coin in which case we use the effect operation raise to raise an exception. Otherwise we return the result of a second coin toss as a string. The two effect operations flip and raise are declared in corresponding effect signatures Amb (for ambiguity) and Exc (for exceptions) in Subfigure 2b.

The type signature of drunkFlip declares that it may use effects by adding the checked exception Effects to its throws clause. It may use effect operations from the effect signatures Amb and Exc because it takes instances of those interfaces as arguments. The drunkFlip method does not rely on any concrete implementation of the effect operations raise and flip since it merely uses the interfaces Amb and Exc in its signature. The caller is free to pick implementations of Amb and Exc, determining the semantics of the effect operations. For example, we could ignore effect handlers (and our EFFEKT library) and use real side-effects to implement Amb and Exc directly. For flip we use a random number generator and for raise we use native exceptions. 


\subsection{Handling Effects}

We now show how to freely mix handlers, if we use effect operations like in Figure 2 and implement handlers for them by using our EFFEKT library. This is an example of the modularity benefits provided by effect handlers. The full implementation of our handlers will be given shortly, for now it is enough to know that we define our handlers as follows:

class Maybe $<R>$ extends Handler $<R$, Optional $<R \gg$ implements Exc $\{\ldots\}$ class AmbList $<R>$ extends Handler $<R$, List $<R \gg$ implements Amb $\{\ldots\}$

Like our first interpretation of Amb and Exc above, the classes Maybe and AmbList again implement the corresponding effect signatures. The handlers extend our library class Handler $<R, E>$ to express that they represent effect handlers. In general, every handler for an effect gives semantics to the corresponding effect operations. It interprets an effectful program which would compute a result of type $R$ (mnemonic for "return type") into a new semantic domain of type $E$, the effect domain. The effect domain is chosen to have enough structure to implement the effect operations. Before looking at the details of how the handlers are implemented, it is instructive to understand how they can be used. This is best explained by analogy to exception handling. Effect handlers are a generalization of exception handlers [Plotkin and Pretnar 2009]: Effect operations generalize throw to other effects while handling an effect operation is a generalization of try $\{\ldots\}$ catch $\{\ldots\}$. In pseudo syntax, handling effect operations like flip and raise can be thought of as:

$\operatorname{try}\{\operatorname{try}\{\ldots$ flip ()$\ldots$ raise $(" . . ") \ldots\}$ catch Exc with Maybe $\}$ catch Amb with AmbList

Like with exception handlers in Java, the try-block describes the dynamic scope of the corresponding handler. In comparison, using EFFEKT, handling effects Amb and Exc looks like:

$$
\begin{aligned}
& \text { List }<\text { Optional }<\text { String } \gg \text { res }_{1}=\text { handle }(\text { new AmbList }<\text { Optional }<\text { String } \gg \text { ) } \text { amb } \rightarrow \\
& \text { handle(new Maybe }<\text { String }>0 \text {, exc } \rightarrow \operatorname{drunkFlip}(\operatorname{amb}, \text { exc })) \text { ); }
\end{aligned}
$$

The lambda, which is passed as body to handle $(h, b o d y)$ represents the dynamic scope in which the handler $h$ can be used. Besides registering the handler $h$ for the dynamic scope, handle also passes the handler unmodified as argument to the body. Thus, in our example the variable amb will be bound to the instance of AmbList created on the same line. Running drunkFlip with both effects handled now yields for res $_{1}$ :

\section{- [Optional["Heads"], Optional["Tails"], Optional.empty]}

We can easily swap the two handlers to obtain a different semantics where an exception leads to a termination of the backtracking search.

$$
\begin{aligned}
\text { Optional }<\text { List }<\text { String } \gg r e s_{2}= & \text { handle }(\text { new Maybe }<\text { List }<\text { String } \gg(), \text { exc } \rightarrow \\
\text { handle }(\text { new AmbList }<\text { String }>0, \text { amb } & \rightarrow \operatorname{drunkFlip}(\text { amb }, \text { exc }))) ;
\end{aligned}
$$

Since at least one of the control paths raises an exception, running drunkFlip with the effects handled in the different order yields for $\operatorname{res}_{2}$ :

- Optional.empty

This shows the power of effect handlers: effectful programs can be written fully agnostic of both the semantic domain into which the effects will be interpreted, as well as the order in which the effects will be handled. 


\subsection{Implementing Effect Handlers}

Having seen how effects can be handled, we will now take a closer look at how handlers are implemented. Subfigure $3 \mathrm{~b}$ shows the Handler interface which is relevant for implementing effect handlers. It also shows the type of effectful functions $E f f<S, T>$ which is just like the Java function interface Function $\langle S, T>$ but with its single abstract method being marked as throwing Effects. Similarly, interface $\mathrm{CPS}<A, E>$ corresponds to the nested effectful function type $\mathrm{Eff}<\mathrm{Eff}<A, E>, E>$.

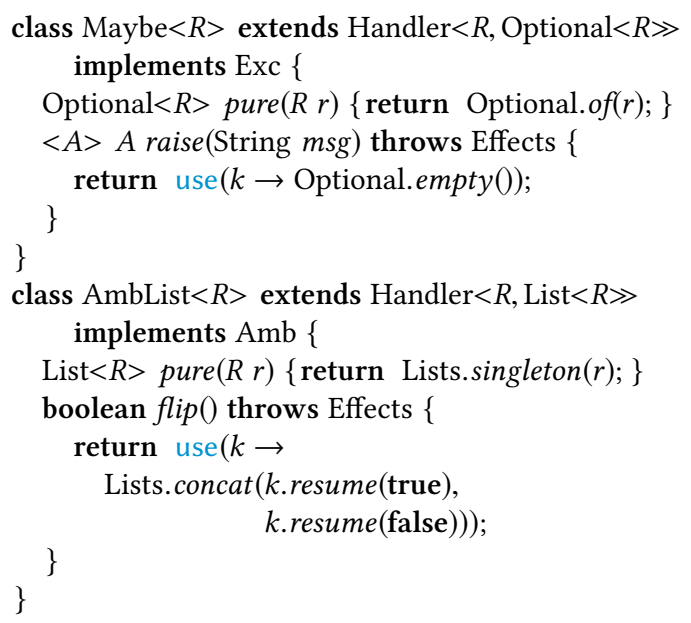

(a) The two effect handlers Maybe and AmbList utilizing use to capture the continuation. abstract class Handler $<R, E>\{$

$E$ pure $(R r)$ throws Effects;

$<A>A$ use $(\mathrm{CPS}<A, E>b o d y)$ throws Effects \{

\}

static $<R, E, H$ extends Handler $<R, E \gg E \hookleftarrow$

handle $(H h, \mathrm{Eff}<H, R>p)$ throws Effects \{

\}

\}

interface $\mathrm{Eff}<S, T>\{$

$T$ resume $(S$ value $)$ throws Effects;

\}

interface $\mathrm{CPS}<A, E>\{$

E apply $(\mathrm{Eff}<A, E>k)$ throws Effects; \}

(b) Interface of the library class Handler and the necessary functional interfaces.

Fig. 3. Implementation of effect handlers for Exc and Amb using the library class Handler .

Every handler needs to implement the abstract method pure to specify how handled programs that don't use the corresponding effect are lifted from $R$ into the effect domain $E$. Additionally, a handler needs to implement all the effect operations which are specified in the effect signature. To implement the effect operations, the handlers are able to utilize the instance method use provided by the library class Handler. Calling this.use(body) in the implementation of an effect operation captures the continuation $k$ and passes it to the provided body. It then continues executing $\operatorname{body}(k)$, effectively passing control to the handler. This allows the handler to suspend and resume the handled effectful program.

Both handlers, Maybe and AmbList implement their effect operations in terms of use (Figure 3a). The handler Maybe, captures the continuation and deliberately discards it in order to implement the raise effect. This mimics the behavior of native exceptions, where an exception causes the unwinding (and discarding) of the runtime stack up to the corresponding exception "handler" (hence the terminology). The handler for ambiguity, in turn, captures the continuation and invokes it twice. Once with true and once with false, each yielding a list of possible results. Finally, it concatenates the two lists ${ }^{7}$. It is important to stress that this is only possible because the continuation captured by use is delimited by the corresponding call to handle. In the implementation of flip, the continuation captured by use will return a list because it is delimited by the call to handle(new AmbList $<$ String $>(), \ldots$ ) and the handler AmbList defines

\footnotetext{
${ }^{7}$ For this example, we assume lists to be immutable and only be constructed by singleton and concat.
} 
the effect domain to have the type List $<$ String $>$. In general, the type of the captured continuation is $E f f<A, E>$. That is, it is an effectful function from $A$ to $E$. Similarly, in raise it is safe to discard the continuation and immediately return Optional.empty because the caller of handle (new Maybe $<$ String, Optional $<$ String $\gg(), \ldots)$ expects a value of type Optional $<$ String $>$.

\subsection{Design of the EFFEKт Library}

We designed EFFEKT as a library for Java around object oriented idioms like subclassing and dynamic dispatch. In summary, the EFFEKT library is based on the following design decisions. Effect signatures are interfaces and handlers are classes implementing the interfaces. We establish a handler passing style where users explicitly pass handler instances to functions that use effect operations. As we discuss in Section 6, this corresponds to a shallow embedding of effect handlers and is similar to the use of type classes by Kammar et al. [2013] and implicits by Brachthäuser and Schuster [2017]. Handler implementations capture the continuation by explicitly calling use. In EFFEKT, tail resumptive handler implementations which exclusively invoke the continuation in tail position can be expressed as simple methods that do not capture the continuation. EFFEKT is a library and neither changes the language nor the type system. Effectful functions always need to be marked to throw an Effects exception, since only marked methods are transformed by our CPS transformation. The exception should never be caught or otherwise suppressed. Handlers and the operations use and handle are designed to not require an advanced type system. In particular, they do not require type constructor polymorphism. EFFEKT does not establish a full static effect typing discipline. Users need to make sure that an effect handler is only used in the dynamic extent of a corresponding call to handle. Calling use directly, or indirectly via some effect operation on a handler outside of the handler scope will result in a runtime error.

\section{IMPLEMENTING AN EFFECT HANDLER LIBRARY IN JAVA IN THREE STEPS}

As can be seen from our running example, programming with effect handlers in EFFEKT is almost just standard Java programming. Only the control operator use and its counterpart handle make the difference in expressivity. This section describes how these control operators can be implemented, bottom up. We start with a CPS translation, that rewrites all methods annotated with throws Effects, build a library for delimited continuations upon the translation and finally implement effect handlers in terms of delimited continuations.

\subsection{Type Selective CPS Transformation by Example}

To support accessing the continuation with use, the EFFEKT framework performs a type selective CPS transformation by instrumenting (that is, rewriting) JVM bytecode. This can either be achieved by hooking into the class loading mechanisms of Java and injecting the transformation at runtime when a class is loaded or by a separate preprocessing phase that rewrites the class files once ("ahead of time"). Implementing the transformation on the level of JVM bytecode opens up the opportunity of reuse for other JVM languages.

While the implementation of EFFEKT rewrites JVM bytecode, for easier accessibility of the paper this section presents the CPS transformation as a semantically equivalent ${ }^{8}$ source-to-source rewriting of the example program drunkFlip. This section provides an overview, Section 5 describes the implementation of the bytecode transformation in more detail and explains how we treat control flow and exceptions.

\footnotetext{
${ }^{8}$ For the example presented in this section, we manually verified that the bytecode of the source-to-source transformation is equivalent to the result of the bytecode transformation (modulo some superfluous register stores/loads).
} 


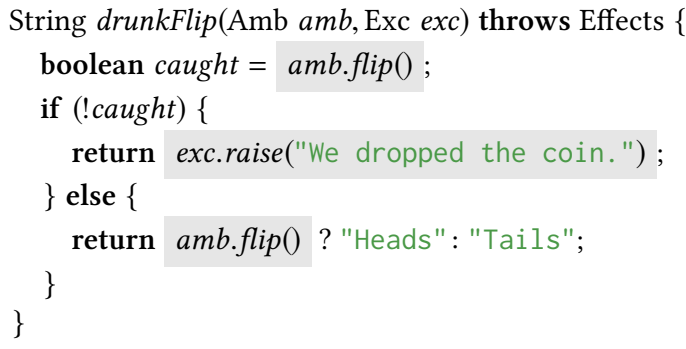

(a) Source method with highlighted effect calls. The effect call to exc.raise is a tail call and does not require an entrypoint.

String drunkFlip(Amb amb, Exc exc) throws Effects \{ Effekt.push $\left(0 \rightarrow \operatorname{drunkFlip}_{0}(a m b\right.$, exc $\left.)\right)$; return null; \}

(b) Generated method stub, only pushing the initial entrypoint.

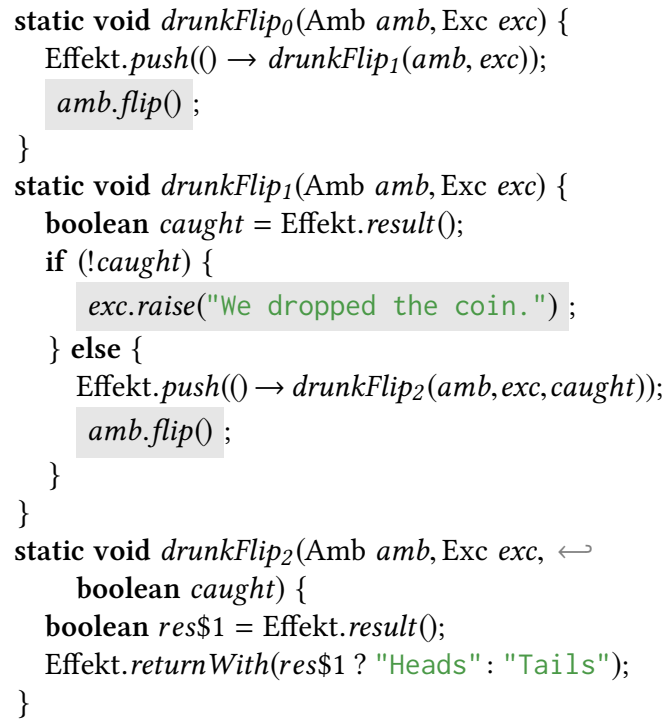

(c) Entrypoints as separate, static method.

Fig. 4. CPS translation of the example in Figure 4a, presented as a source-to-source transformation.

Figures $4 \mathrm{~b}$ and $4 \mathrm{c}$ show the result of transforming the method drunkFlip. We instrument only effectful methods and identify those by means of the throws Effects annotation. Using Reynolds [1972] terminology, we only consider methods marked with throws Effects to be "serious". All other functions are "trivial" and don't require any instrumentation. Consequently, we also only instrument call sites of effectful functions (effect calls). In drunkFlip there are three such effect calls, two to flip and one to raise. We exclude tail effect calls from the translation, that is effect calls immediately followed by a return. For drunkFlip this means that we instrument the two flip calls, since the call to raise is in tail position. We call the code immediately following an effect call an entrypoint. We also treat the initial entrypoint of a function as an entrypoint in this sense.

Similar to Prokopec and Liu [2018], for each entrypoint in an effectful method, we generate a separate entrypoint method. For our example, these are the methods drunkFlipo (the initial entrypoint method), drunkFlip ${ }_{1}$ and drunkFlip d $_{2}$ (corresponding to the two invocations of flip). Entrypoint methods take the function local state as arguments. That is, all local variables and values on the operand stack needed to resume the function execution after the effect call would return.

Similarly to how the JVM would push a stack frame before a method call, we rewrite every effect call to first push a continuation frame to a global user-level stack by invoking Effekt.push Class Effekt has a global static field Effekt.stack that implements the interface Stack shown in Figure 5. For notational convenience, we write Effekt.push instead of Effekt.stack.push. The interface Stack contains all necessary methods used by the instrumented bytecode. A continuation frame is an instance of the Frame interface, also shown in Figure 5. We use Java 8 lambdas to create instances of the Frame interface. The lambdas close over the function local state which they will pass to the entrypoint methods when invoked with enter. Conceptually, we thus represent continuation frames as instances of classes that have one field for each local they store. After pushing the continuation frame, the entrypoint methods call the effectful method and immediately return. Thus, all effect calls in the translated program are tail calls. 
To actually start execution, we call Effekt.trampoline which will continue to pop and enter frames until the stack is empty. If an exception is raised, the trampoline will unwind the user-level stack frame by frame. Each frame starts with a call to result, reraising the exception after restoring the method state. Section 5 shows more details on how we deal with exceptions.

\subsection{Delimited Continuations}

A program that has been transformed with our CPS translation still uses the JVM stack for noneffectful calls but uses our user-level stack for effectful function calls. Different implementations of the interface Stack with different representations also give rise to different additional operations that exploit the corresponding stack representation. In consequence, effectful programs that are executed against a particular stack implementation can make use of those additional operations. In this section, we will develop one particular implementation of Stack that implements additional operations to capture delimited continuations. All code in the rest of this paper is subject to the CPS bytecode transformation and all methods annotated with throws Effects will be instrumented.

As we will see in subsection 3.3, the effect handler library can be implemented as a very thin layer on top of multi-prompt delimited continuations [Brachthäuser and Schuster 2017; Kiselyov and Sivaramakrishnan 2016]. Our implementation of multi-prompt delimited continuations closely follows Dyvbig et al. [2007], but translated to Java and to our setting of bytecode instrumentation. We extend the Stack implementation sketched above and implement two additional methods pushPrompt and withSubcont as summarized by the following interface:

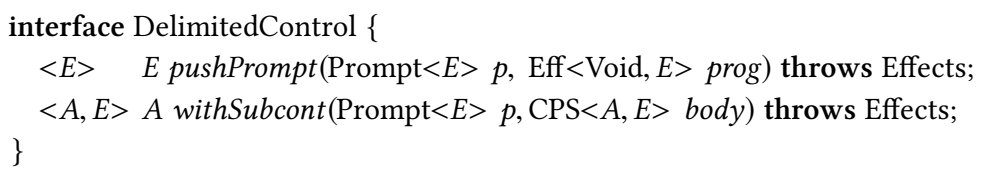

Instances of Prompt $<E>$ (interface defined in Figure 6c) are used to mark positions on the stack. The type of an effectful function Eff and of effectful programs that use an effectful continuation CPS have been defined in Figure 3b. ${ }^{9}$ The type parameter $E$ of Prompt unifies with the type of the computation that we delimit with pushPrompt. Capturing a continuation with withSubcont, the return type of the continuation and of the body have to match the type of the prompt $E$.

3.2.1 Using Delimited Continuations. Assuming that the global stack instance supports the methods from DelimitedControl, for any two different prompts $p_{1}, p_{2}$ : Prompt $<$ Integer $>$ we can implement examples such as:

$2 *$ Effekt.pushPrompt $\left(p_{1}, 0 \rightarrow 1+\right.$ Effekt.withSubcont $\left(p_{1}, k \rightarrow k\right.$.resume $(2) * k$.resume $\left.\left.(6)\right)\right)$;

- $2 *((1+2) *(1+6))=42$

The continuation $k$ corresponds to the evaluation context $1+\square$, since it is delimited by $p_{1}$. We invoke it twice. A second example illustrates prompt search and discarding of the continuation:

$2 *$ Effekt.pushPrompt $\left(p_{1},() \rightarrow 1+\right.$ Effekt.pushPrompt $\left(p_{2},() \rightarrow 3 *\right.$ Effekt.withSubcont $\left.\left.\left(p_{1}, k \rightarrow 21\right)\right)\right)$;

- $2 * 21=42$

The captured continuation $k$ contains the program segment marked by prompt $p_{1}$. It corresponds to the evaluation context $\left.1+\operatorname{pushPrompt}\left(p_{2},() \rightarrow 3 * \square\right)\right)$. We discard the continuation and replace it by the value 21 .

\footnotetext{
${ }^{9}$ When effectful functions don't require an argument we will use $\mathrm{Eff}<$ Void, $A>$. To avoid materializing instances of Void and binding them, we write $f$.resume () as a short hand for $f$.resume(null) and $(0 \rightarrow \ldots)$ instead of (unusedVoid $\rightarrow \ldots)$.
} 


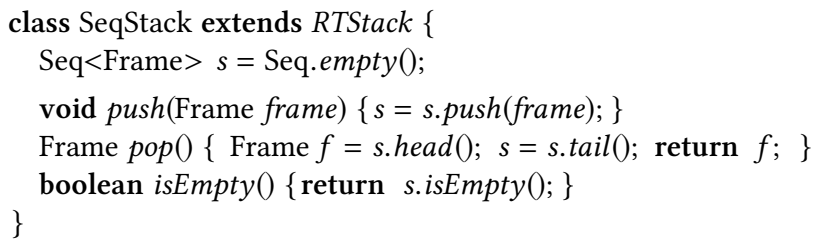

(a) Implementation of Stack, forwarding to an immutable stack.

class DelimCC extends SeqStack implements DelimitedControl \{ $<E>E$ pushPrompt (Prompt $<E>$ p, Eff $<$ Void, E $>$ prog) throws Effects \{ $s=s \cdot \operatorname{mark}(p)$; return prog.resume $)$; \}

(b) Implementation of delimited control in terms of an immutable, splittable stack: Seq. Usage of Seq is highlighted. interface Prompt $<E>\{\}$

(c) Interface Prompt, used to mark stack segments. Prompts are compared with reference equality.

(d) Immutable stack that allows marking and splitting at positions $p$.

Fig. 6. Implementation of control operators to capture delimited continuations.

3.2.2 A Splittable Stack Implementation. The simplest implementation of Stack that comes to mind is to store a list of frames in a field $s$ and implement all abstract operations of Stack by forwarding to this list $s$. Figure 6a drafts such an implementation. For now we just assume Seq to be an immutable implementation of a stack data structure with elements of type $A$. While very simple, running a program with this stack implementation already has the benefit that it performs trampolining and thus reduces JVM stack usage, which might avoid stack overflows. However, the real power of the translation comes from the fact that Stack implementations can add new methods which expose additional (control) operators. To implement the additional control operators pushPrompt and withSubcont we need to mark positions on the runtime stack (mark), slice the stack at given positions ( before, after) and prepend whole stack segments (prependTo) [Dyvbig et al. 2007]. The stack data structure Seq (Figure 6d) that we have already used above offers exactly these operations. One can think of $\mathrm{Seq}<A>$ as a two-sorted stack that contains elements of type $A$ and markers of type Prompt. Calling s.before( $p)$ returns the initial segment up to, but not including the first occurrence of the marker $p$. This segment contains all the recently pushed elements after $p$ has been pushed. Calling s.after $(p)$ returns the remainder of the stack. This segment contains all the elements which have been pushed before the marker $p$ has been pushed. This is characterized by the following equation:

s.before $(p) \cdot \operatorname{prependTo}(s . \operatorname{after}(p) \cdot \operatorname{mark}(p)) \equiv s \quad$ where $p \notin s$. before $(p)$ 
3.2.3 Pushing a Prompt. Figure 6b shows the implementation of the DelimitedControl interface, using Seq. To implement pushPrompt, we mark the stack using the provided prompt $p$ and update the mutable reference $s$ with the now marked stack. We then resume with the effectful program prog. Being effectful, the program prog pushes additional frames onto the stack. We can capture those frames later by slicing the stack at the position of the installed marker $p$.

3.2.4 Capturing a Continuation. The control operator withSubcont $(p, b o d y)$ captures the continuation $k$ up to the next dynamically enclosing $\operatorname{push\operatorname {Prompt}}(p, \ldots)$ and conceptually replaces the call to pushPrompt with a call to body.apply $(k)$. Its implementation in Figure 6b stores all frames that have been pushed after $p$ in a local variable init. This segment corresponds to the delimited continuation from type $A$ to type $E$. That is, the top most frame expects Effekt.result to return a value of type $A$. The initial segment and the prompt marker are then removed from the stack by mutating it with $s=s$.after $(p)$. This leaves a segment on the stack which expects a value of type $E$ to continue program execution. The continuation $k$ implements the functional interface Eff with method resume() throws Effects. It closes over the initial stack segment init and, when invoked, prepends it to the stack $s$. This implements the desired semantics of resuming the delimited continuation: The runtime system will first run the initial stack segment init before it eventually continues at the callsite of resume within body.

There are two casts involved, that require some explanation. Both withSubcont and the continuation $k$ are effectful. Thus the respective caller will be instrumented. However, by mutating field $s$ and modifying the stack, we change the execution context. In withSubcont we remove the initial segment of the stack and thus the new caller expects a value of type $E$ not $A$. In the continuation we prepend the initial segment and thus the caller now expects an $A$ not $E$. The Java typechecker is ignorant of our transformation and the modifications to our own callstack. Hence the casts ${ }^{10}$.

\subsection{Implementation of the EFFEкт Library}

In the previous subsections we have seen how programs which contain throws Effects annotations are CPS translated. We extended the runtime environment in which those translated programs are executed to support multi-prompt delimited continuations. Equipped with multi-prompt delimited continuations we are now finally ready to see how the effect handler library can be implemented.

The expressive power of effect handlers comes from the two operations handle and use which are encapsulated in the library class Handler. Figure 7 shows the implementation of these two operations in terms of delimited continuations. We use handlers themselves as prompt markers. An effect handler with effect domain $E$ implements Prompt $<E>$. The answer type of delimited continuations thus will be the effect domain $E$. In the implementation of handle we push the handler as a prompt before resuming with prog. The pushed handler will delimit the extent of continuations captured by that handler. By calling h.pure after resuming, programs that don't use effects will be lifted from $R$ to $E$. The method use calls withSubcont with the current handler instance this as a prompt marker to capture the continuation up to the most recent call to handle on this handler instance. It then passes the captured continuation $k$ to body.

Effect handlers in EFFEKT are deep handlers [Kammar et al. 2013]. That is, all effect operations are recursively handled by the same handler. To implement deep handlers, we modify the continuation to re-push the prompt before resuming to make sure that all subsequent calls to use on this handler are again delimited by this. That is, both continuations $k_{1}$ and $k_{2}$ in handle $\left(h, 0 \rightarrow 1+h\right.$.use $\left(k_{1} \rightarrow \ldots\right)+h$.use $\left.\left(k_{2} \rightarrow \ldots\right)\right)$ should be delimited by handle $(h, 0 \rightarrow \square)$ Our operations handle and use are thus conceptually very similar to spawn and the corresponding controller by Hieb and Dybvig [1990].

\footnotetext{
${ }^{10}$ Since $A$ and $E$ are generic type parameters, they will nevertheless be erased and the program can safely be executed.
} 


\subsection{Case Study: Cooperative Multitasking}

Like generators and coroutines, cooperative multitasking and async/await can be implemented as a library [Dolan et al. 2017; Leijen 2017b]. Programs can use the Coop effect to fork and suspend processes. A process is an effectful program Eff $<$ Void, Void $>$. The RoundRobin handler ${ }^{11}$ in Figure 8 implements a scheduler that keeps a queue of all running processes in its handler state. Forking a process $p$ is implemented by using flip to get a boolean and then either starting $p$ and discarding the continuation when $p$ returns or continuing as normal otherwise.

Yielding is implemented by enqueueing the continuation of the process and immediately returning. The handler will then pick the next process to execute. A program that uses Coop is executed by first handling the effect using RoundRobin and then running the scheduler with run.

\section{IMPLEMENTATION OF THE TYPE SELECTIVE CPS TRANSFORMATION}

Section 3.1 illustrated the type-selective CPS translation by example as a source-to-source transformation. This section presents the implementation of the translation on the level of bytecode and shows how we handle control flow elements like jumps and exceptions. The translation is interesting in that it uses Java 8 closures to create continuations. This is in contrast to other bytecode translations which we will compare in Section 6.1.

We implemented the transformation described in this section using the OPAL framework ${ }^{12}$ by Eichberg and Hermann [2014] for static analysis and synthesis of JVM bytecode. Our implementation of the CPS transformation as well as all other components of EFFEKT can be found online ${ }^{13}$.

We minimize the presentation to a relevant core set of language features, leaving out details that distract from the essence of the transformation: Storing all necessary function local state in closures and inserting calls to the EFFEKT API as described in Section 3. We model the JVM and thus assume an abstract machine with registers (also referred to as locals, since they are function local), an operand stack (also referred to as operands, again function local) and a frame stack (commonly referred to as stack). We use the term function local state to refer to the values stored in locals and operands at a given time in the execution of a method. We also assume the JVM calling convention that function arguments are pushed on the operand stack by the caller, but accessible as locals by the callee, starting from register index 0 . We assume every bytecode instruction is labeled, but omit labels that we never refer to.

We explain the transformation on an effectful example method doLoop:

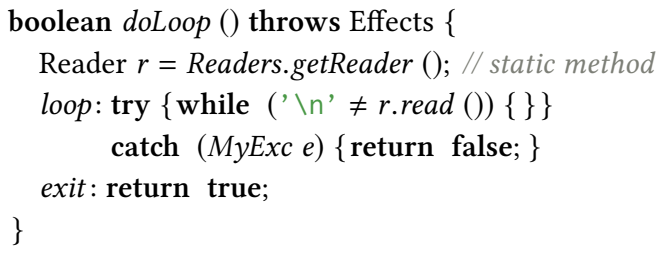

The bytecode of the method doLoop is shown in Figure 9a. The method doLoop will perform effect calls to r.read () until the result is either a newline or r.read () raises a native exception. The example includes exception handling to illustrate in more detail how the bytecode instrumentation interacts with native exceptions. As in the JVM, exception-handling is modeled external to the list of bytecode instructions of a method and exception handlers are given in the form of regions as

excregion tryStartLabel tryEndLabel catchLabel exception

\footnotetext{
${ }^{11}$ The type parameters of Handler are Void, they are omitted due to lack of space. pure just returns null .

${ }^{12} \mathrm{http}: / /$ www.opal-project.de/

${ }^{13}$ http://github.com/b-studios/jvm-effekt
} 
In our example, let us assume an exception region excregion loop break catch MyExc is in place. That is, if an exception of type MyExc is raised in the dynamic region between loop and break execution will be continued at catch .

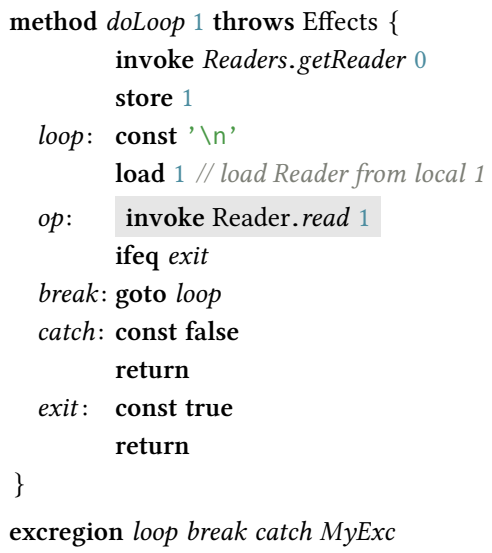

(a) Bytecode of method doLoop .

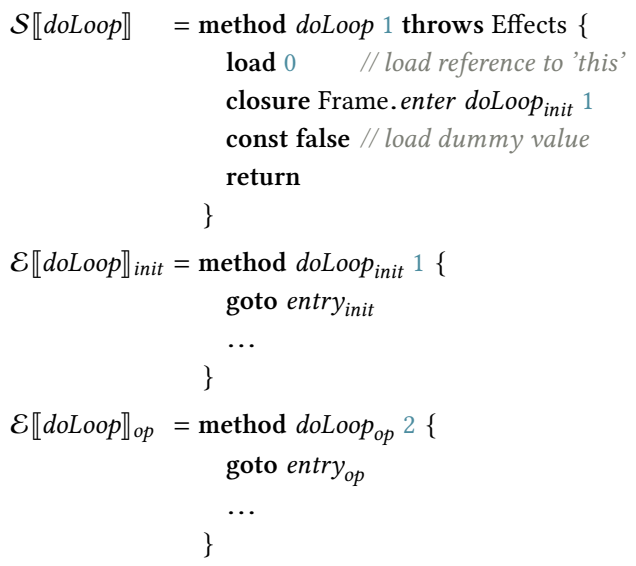

(b) Generated methods - method bodies in Fig. 9d. effCalls $_{\text {doLoop }}=[$ init, op $]$ operands $_{\text {init }}=0$ operand $s_{\text {op }}=1$ tmpLocal $_{\text {doLoop }}=2$ locals sinit $_{1}=[0]$ local $_{\text {op }}=[1]$

(c) Meta information as obtained by static analysis.

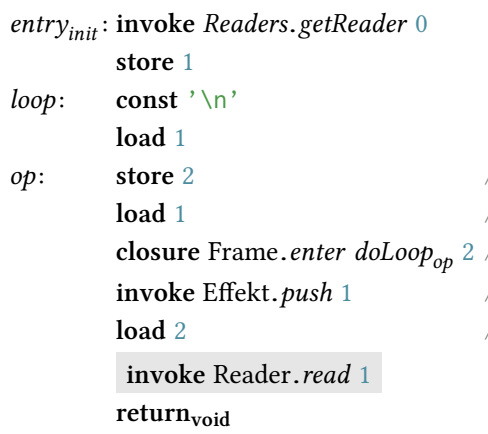

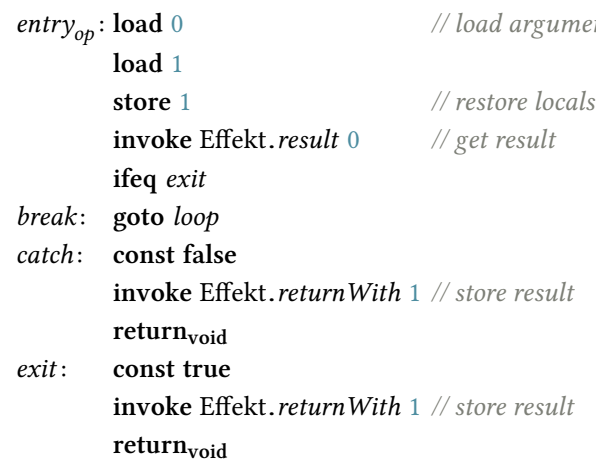

(d) Result of translating the instructions of method doLoop .

Fig. 9. Example of translating a method doLoop.

The syntax of the term-language is summarized in Figure 10a. For simplicity of the presentation, we do not concern ourselves with types and thus choose a uni-typed term-language. The translation does not distinguish instance methods and static methods. Hence, we only include a single method definition that consists of a name, a list of exception handler regions (the exception table), a list of potentially raised exceptions and a list of labeled instructions. The syntax of bytecode instructions Instr in Figure 10a includes instructions to load constant values (const $v$ ) to the operand stack, instructions to load from and store into function local registers (load index, store index) and control flow instructions ( return, throw, ifeq ${ }^{14}$ and goto ). Finally, as with method declarations,

\footnotetext{
${ }^{14}$ For our example, we assume ifeq label pops two values and jumps to the given label if the two values are equal.
} 
As was seen in the source-to-source transformation in Figure 4, for one effectful method $m$, we generate multiple methods: a single method stub, using the translation function $\mathcal{S} \llbracket \cdot \rrbracket$ and one entrypoint method for each effect call at label eff using the translation function $\mathcal{E} \llbracket \cdot \rrbracket_{\text {eff }}$ (both translation functions are defined in Figure 10b). Every effect call eff : invoke fun arity inside a given method $m$ gives rise to an entrypoint uniquely identified by the label eff. The entrypoint

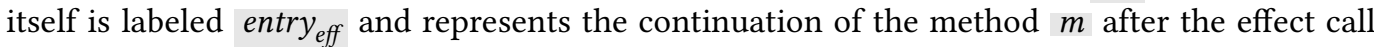
returned. For consistency, we also treat the initial entrypoint at label init as effect call. The special entrypoint entry $y_{\text {init }}$ refers to the label of the first original instruction of the method. By explicitly pushing the initial entrypoint, we perform trampolining for each effect call. For the method doLoop, we thus generate three methods: the method stub doLoop and the two entrypoint methods doLoop init $_{\text {and }}$ andoop op (Figure 9b).

Rule T-STUв generates the method stub that first saves the local state and then immediately returns a dummy value. At that point, the local state only consists of the arguments supplied to the function call. As we will see shortly, saveState thus pushes a closure that closes over the call arguments and resumes with method doLoop $p_{\text {init }}$ when invoked. The returned result of the stub method will never be used, hence loadDummyResult can load any constant value.

Rule T-EnTRYPOINT generates two static entrypoint methods doLoop $p_{\text {init }}$ and doLoop op $_{\text {. The }}$ bodies of the two methods are exactly the same after the initial goto instruction and are given in Figure $9 \mathrm{~d}$. The only difference is the initial jump. Since generating almost identical methods for each entrypoint leads to unnecessary growth of the class file, in our implementation of EFFEKT, we perform dead code elimination after generating the bytecode. Using closures to save state, only saving live variables and performing dead code elimination ultimately results in code which is very close to handwritten code in continuation passing style (as in Figure 4c).

\subsection{Saving Function Local State}

To generate state saving and restoring code, we use the following information about a method $m$, which we obtain by static analysis:

- the set of all labels corresponding to effect calls, also including the first label entry $y_{\text {init }}$ but no effect tail calls - effCalls $(m) \in \overline{\mathbb{L}}$,

- the index of the first free local register, not used by the original instructions of method $m$ tmpLocal $_{m} \in \mathbb{N}$.

Likewise, for each effect call eff in a method $m$, the transformation uses the following information which we again obtain by static analysis:

- the number of operands on the operand stack after the effect call (not including the result of the effect call) - operands eff $_{\in} \in \mathbb{N}$,

- the list of indices of local registers which are alive after the effect call - locals $_{\text {eff }} \in \overline{\mathbb{N}}$,

For method doLoop, the static information is given in Figure 9c. We also define closureArity eff $^{\text {to }}$ equal operands $s_{\text {eff }}+\mid$ locals $_{\text {eff }} \mid$, referring to the total number of values that need to be stored in the closure, that is all operands and the number of locals which are live after the effect call. To actually save the state, the meta function saveState generates code that stores those parts of the function local state which are necessary to resume the execution of the function. This includes all operands (after the effect call) and the contents of all registers which correspond to live local variables. This is achieved in three steps:

(1) all live local variables are loaded to the operand stack; the operands do not need to be loaded since they already are on the operand stack

(2) a new instance of a Frame is created as a lambda using the given method name eff as the body of the lambda and closing over closureArity $_{\text {eff }}$-many values on the operand stack; 
(3) finally, the newly created frame is pushed using Effekt.push.

In JVM bytecode, closures are created by issuing a specific invokedynamic call to a lambda metafactory. We refer to this call only in its specialized form as

closure interfaceName name arity

The call to closure is provided with a name $\in$ Name of a method which serves as the implementation of the lambda, implementing a single-abstract-method interface interfaceName $\in$ Name ${ }^{15}$ and an arity $\in \mathbb{N}$ which specifies the number of values the lambda should close over. The JVM runtime passes the closed-over values as additional arguments to the implementing method when the closure is applied.

\subsection{Translation of Instructions}

To generate the bodies of entrypoint methods, Figure 10c defines the semantic function $\mathcal{I} \llbracket \cdot \rrbracket$ which specifies how labeled instructions are translated. The result of translating the body of doLoop can be found in Figure 9d. Figure 10d gives the implementation of some of the helper functions. They are meta functions and thus expand at translation time to generated code. Given a list of register indices, the unlisted functions loadLocals and storeLocals generate bytecode that loads from (respectively stores to) all given locals. We use the notation $x$ to $y$ to denote a range of indexes from $x$ to $y$, both ends inclusive. Similarly, $x$ downTo $y$ denotes a decreasing range.

The translation of bytecode instructions behaves as identity (Rule T-OTHER) except for effect calls (Rule T-INVOKE-EFF) and returns (Rule T-RETURN ). To stress, non-effectful calls don't require any modification. To translate effect calls, rule T-INVOKE-EFF saves the function local state, performs the effect call and then suspends the method by returning to the trampoline ( return $\left._{\text {void }}\right)$. In the translated program, all jumps to the effect call within $m$ should point to the instrumented call instead. Therefore we change the label eff to point to the first instruction of the state saving code. This automatically also affects exception regions that mention eff. As in our doLoop example, the effect call might require arguments that reside on the operand stack at the time of state-saving. To account for this, we use temporary locals (which will not be stored in the closure) to set the call operands temporarily aside.

The remainder of the function after the effect call is labeled with entry $y_{\text {eff }}$. Since it immediately follows a return, this part of the code is only reachable by the goto entry eff in the corresponding entrypoint method. At that time, all function state necessary for resumption has been passed as arguments and is thus stored in first closureArity $_{\text {eff }}$-many registers. For our example of doLoop,

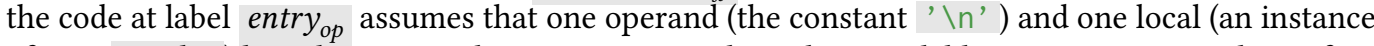
of type Reader ) have been passed as arguments and are thus available via registers 0 and 1 . Before the function can be resumed, its state needs to be reset to where it has been left off. The meta function restoreState loads all saved operands and locals (in this order) to the operand stack. It then writes the locals to the correct registers (in reverse order). The result of the previous effect call is obtained by Effekt.result. If the previous effect call exited abnormally by throwing an exception, Effekt.result as implemented in Figure 5, will re-raise this exception. Since we already restored all operands and locals, the exception will be raised in the correct context and trigger the correct exception handlers. Being defined in terms of labels, our translation does not need to modify the exception table. The rule T-RETURN replaces every return with a call to Effekt.return With to install the second half of the special calling convention.

${ }^{15}$ For the translation, interfaceName will always be Frame.enter 


\section{DISCUSSION AND RELATED WORK}

We discuss design decisions, related work, performance and future work. Existing implementations of libraries and languages for (algebraic) effect handlers are either translations to a high level language or involve a custom runtime implementation. High level implementations translate effect handlers into delimited continuations [Brachthäuser and Schuster 2017; Kammar et al. 2013; Kiselyov and Sivaramakrishnan 2016], free monads [Kiselyov and Ishii 2015] or perform a source to source CPS translation into another high-level language [Hillerström et al. 2017; Leijen 2017c]. Other implementations require a custom runtime that supports stack manipulation [Bauer and Pretnar 2015; Dolan et al. 2017] or setjump / longjump [Leijen 2017a]. In this paper, we explore a new implementation technique for effect handlers in terms of a CPS transformation of bytecode. The discussion is split accordingly into a discussion of the bytecode transformation and a discussion of the EFFEKT framework for programming with effect handlers as a whole.

\subsection{Continuations on the Java Virtual Machine}

We review related work on (delimited) continuations and CPS transformations in the context of the Java virtual machine. While implementations that modify the JVM exist [Dragos et al. 2007; Stadler et al. 2009] or are under development [Pressler 2017]. While those specialized runtimes could potentially be used as backend for our effect handler library, here we will focus on library solutions. We compare our CPS transformation with three other Java projects that perform bytecode instrumentation. A library for fibers "Quasar" ${ }^{16}$, a library for one-shot continuations "JavaFlow"17 and a library for coroutines ${ }^{18}$.

Continuation instantiation. Approaches to capture the continuation can be characterized by the point in time the continuation is constructed. CPS transformations create the continuation before the execution of an effectful call. The continuation is thus always immediately available. This is how we implemented EFFEKT. It is also the case for implementations of effect handlers that rely on CPS [Hillerström et al. 2017] and corresponding monadic implementations in eager languages like Scala Effekt [Brachthäuser and Schuster 2017]. Quasar also explicitly stores all function state before entering an effect call. Another approach is to instantiate the continuation only when it is needed. Typically, to signal that the continuation needs to be captured, the effectful function can use a special exception [Loitsch 2007; Pettyjohn et al. 2005; Sekiguchi et al. 2001], sum types [Kiselyov and Sivaramakrishnan 2016] or global flags (JavaFlow, Coroutines).

Stack Restoration. Some implementations are designed to fully restore JVM stack when a continuation is resumed (Quasar, JavaFlow). This simplifies integration with exceptions, stack traces and debuggers. Full restoration of the stack is a technical consequence of not having a first class representation of continuation frames. In consequence, all bytecode continuation libraries in Java that we are aware of resume a continuation by replaying all function calls. However, restoring the stack is always linear in the depth of the stack since all function calls need to be replayed. ${ }^{19}$ In contrast to that, in EFFEKT we explicitly reify each continuation frame as a closure and upon resumption we enter the first frame without restoring the Java stack. While this helps to reduce the asymptotic complexity from quadratic to linear, stack traces in EFFEKT only show very few frames which can impede debugging.

\footnotetext{
$\overline{{ }^{16} \mathrm{http} / / / \text { docs.paralleluniverse.co/quasar }}$

${ }^{17} \mathrm{http}$ ://commons.apache.org/sandbox/commons-javaflow/

${ }^{18} \mathrm{https} / /$ github.com/offbynull/coroutines

${ }^{19}$ For Quasar, this observation has also been made by Aleksandar Prokopec (Oracle Labs) - private communication.
} 
Function state representation. The state necessary to resume a suspended function consists of the function local state and an entrypoint label. It can be represented and stored in different ways. The entrypoint label can be encoded as a number that will be dispatched upon with a switch statement at the beginning of the method. This is commonly combined with storage of the function local data in a stack like data structure (Quasar, JavaFlow, Sekiguchi et al. [2001], Bierman et al. [2012]). An alternative is to replace the switch by dynamic dispatch and to store the function data in a closure [Pettyjohn et al. 2005]. This is how EFFEKT is implemented.

Multiple resumptions. We designed EFFEKT in a way that continuations can naturally be resumed multiple times. In implementations supporting only one-shot continuations, state update can be destructive, which makes it easier to implement continuations efficiently [Dolan et al. 2015]. While EFFEKT maintains one global immutable runtime stack (as in Figure Figure 5), Quasar and JavaFlow maintain one mutable stack per delimited continuation / fiber. In such a setting, multiple resumptions are implemented by deeply cloning the corresponding stack and all nested stacks before resuming In EFFEKT function local state is copied into immutable frames. Also stack segments are immutable and can be shared across multiple resumptions.

\subsection{Relation to other (Algebraic) Effect Handler Libraries and Languages}

Most implementations of libraries and languages for (algebraic) effects are based on a deep embedding of effect operations. They reify effect operations as alternatives in a sum type. For instance, the flip effect operation would be reified as a constructor of an algebraic data type Amb. Handlers then use pattern matching to interpret the reified effect operations [Bauer and Pretnar 2015; Hillerström et al. 2017; Kiselyov and Ishii 2015; Kiselyov and Sivaramakrishnan 2016; Leijen 2014]. To mix programs with different effects means to extend an open union type of reified effect operations.

In contrast, EFFEKT builds on a shallow embedding [Carette et al. 2007; Hudak 1998] of effect operations. Shallow embeddings can be structured in a pleasingly extensible way [Oliveira and Cook 2012]. Interpretation of effect operations is moved from (external) pattern matching to (internal) dynamic dispatch which makes a shallow embedding of effect operations a good fit for object oriented programming languages. Kammar et al. [2013] base their library implementation of algebraic effect handlers on Haskell type classes, effectively performing a shallow embedding. Using type classes helps Kammar et al. to achieve good performance results since it prevents the materialization of constructors for effect operations. Brachthäuser and Schuster [2017] present a monadic library implementation of effect handlers for Scala using implicit parameters and handler passing. They show how programming with algebraic effects is an instance of the expression problem and explore how shallow embedded handlers open up different dimensions of extensiblity.

One advantage of the shallow embedding is that it simplifies typing. We use dynamic dispatch instead of implementing a pattern matching interpreter. This helps us to avoid advanced typing features, such as type constructor polymorphism [Kiselyov et al. 2013] or generalized algebraic data types [Kiselyov and Ishii 2015].

Combining OO with effect handlers, we define use as a method on Handler. As a method, it naturally shares the type of the effect domain with its implementing class. Others require path dependent types for the same purpose [Brachthäuser and Schuster 2017]. In contrast, EFFEKT as presented in this paper is designed to remove requirements on the type system and to blend in with Java programming paradigms. The bytecode instrumentation and the Stateful interface both enable the direct use of mutable state and effect signatures are modeled by simple interfaces (as compared to traits with type members and path dependent types in Scala Effekt).

Leijen [2017a] explicitly tags each effect operation in a handler with information about how the continuation is used to implement important optimizations. Similarly explicit, in EFFEKT, handlers 
Table 1. Performance of bytecode instrumentation implementations. Runtime in ms, lower is better.

\begin{tabular}{llrrrrr}
\hline & \multicolumn{7}{c}{ Time in ms (Confidence Interval) } \\
\cline { 2 - 7 } Benchmark & Baseline & \multicolumn{1}{c}{ EFFE KT } & EFFEKT $_{\text {opt }}$ & Coroutines & Quasar & JavaFlow \\
\hline Stateloop 1M & $1.61 \pm 0.09$ & $29.76 \pm 2.57$ & $1.91 \pm 0.04$ & $5.52 \pm 0.35$ & $69.02 \pm 2.59$ & $14.82 \pm 0.48$ \\
RecursiveOnce 1K & $0.01 \pm 0.0$ & $0.69 \pm 0.22$ & $0.34 \pm 0.01$ & $0.07 \pm 0.0$ & $0.23 \pm 0.03$ & $8.18 \pm 0.19$ \\
RecursiveMany 1K & $0.01 \pm 0.0$ & $1.05 \pm 0.38$ & $0.4 \pm 0.07$ & $10.29 \pm 1.41$ & $68.07 \pm 2.07$ & $3363.74 \pm 23.46$ \\
Skynet 1M & $2.74 \pm 0.03$ & $171.34 \pm 5.55$ & $62.13 \pm 3.87$ & $35.19 \pm 2.51$ & $762.1 \pm 155.95$ & $1277.51 \pm 54.18$ \\
SkynetSuspend 1M & $2.74 \pm 0.03$ & $414.56 \pm 9.2$ & $147.4 \pm 5.44$ & $50.46 \pm 2.95$ & $1113.15 \pm 112.78$ & $7198.72 \pm 122.56$ \\
\hline
\end{tabular}

capture the continuation with use. Tail resumptive handlers don't need to capture the continuation and don't call use.

In other libraries and languages for effect handlers, an effect operation implicitly resolves to the dynamically closest handler implementation. In contrast, we require the user to explicitly select the handler to use. This has the advantage that no confusion arises when multiple handlers for the same effect are present and that we avoid any search for the correct handler implementation in some kind of handler stack. Explicitly selecting which handler to use also avoids the problem of effect encapsulation [Biernacki et al. 2017; Lindley 2018], that is, handlers accidentally handle effects. The disadvantage is that explicit handler selection is more verbose and fragile to changes.

Due to our design decision of a shallow embedding of effect handlers, handlers in EFFEKT are deep handlers [Kammar et al. 2013]. That is, all effect operations in the continuation captured by use will automatically be handled recursively by the very same handler. However, if a shallow handler semantics is required, it can be achieved by reifying the command-response trees of selected effect operations. Similar to the conversion from shallow to deep embedding, a reifying effect handler interprets a program of type $R$ into a free-structure Free $<R>$ [Kiselyov et al. 2013] which then can be interpreted step-by-step. While this encoding is possible, it can lead to performance problems and memory leaks.

\subsection{Performance}

We report on some preliminary performance results. Like the discussion, the evaluation of performance is split into two parts: A part on the CPS transformation and a part on EFFEKT as a library for programming with effect handlers. All benchmarks were executed on a $2.5 \mathrm{GHz}$ Intel Core i7 with $16 \mathrm{~GB}$ of memory using the ScalaMeter, a state of the art JVM benchmarking library.

We also show the performance results for a variant of EFFEKT that implements several optimizations: Continuation frames are not materialized upfront, but only when needed. To avoid push-pop-enter cycles, the initial entrypoint is not explicitly pushed but inlined. Only methods that contain at least one non-tail effect call are instrumented. We refer to this variant as EFFEKT opt $_{\text {. }}$ The user programs using EFFEKT don't need to be changed.

Performance of the Bytecode Instrumentation. We evaluate the performance of our CPS transformation comparing with Quasar 0.7.9, a recently maintained fork of JavaFlow ${ }^{20}$ in version 2.2.1 and Java Coroutines in version 1.4.0. We also measure the overhead compared to a baseline that does not capture continuations. All libraries perform some sort of bytecode transformation to support capturing the continuation. Since each of the libraries targets a particular domain (coroutines / fibers), capturing the continuation also involves additional overhead specific to the target application. Where possible, we reduced this overhead by disabling features - focusing on the

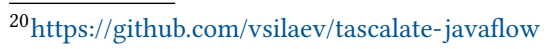


Table 2. Performance of effect libraries. Runtime in ms, lower is better.

\begin{tabular}{lcccc}
\hline & \multicolumn{4}{c}{ Time in ms (Confidence Interval) } \\
\cline { 2 - 5 } Benchmark & EFFEKT & EFFE KT $_{\text {opt }}$ & Scala Effekt & Scala Eff \\
\hline Countdown 10K & $3.35 \pm 0.07$ & $2.47 \pm 0.12$ & $6.07 \pm 0.32$ & $34.39 \pm 2.59$ \\
Countdown8 1K & $1.31 \pm 0.39$ & $1.77 \pm 0.1$ & $2.31 \pm 0.12$ & $36.92 \pm 3.0$ \\
NQueens (10) & $19.5 \pm 0.38$ & $16.09 \pm 0.19$ & $40.95 \pm 0.54$ & $49.89 \pm 2.17$ \\
\hline
\end{tabular}

continuation capturing aspect, only. The results of the measurements can be found in Table 1 . To assess the instrumentation overhead, the Stateloop benchmark counts down from one million to zero, performing some computation work at each step but not capturing the continuation. We can see that most of the overhead of creating continuation frames is eliminated in the alternative

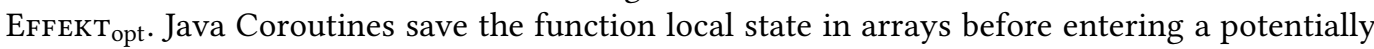
suspending function call. This is unnecessary for the Stateloop benchmark, which does not suspend. To measure performance of capturing the continuation, the RecursiveOnce and RecursiveMany benchmarks also count down, but as recursive functions. For the first benchmark we suspend the computation once before returning the result (at stack-depth 1,000); correspondingly, for the second one we suspend once at every recursive call. Resuming continuations is linear in stack depth for all implementations but the EFFEKT implementations. In consequence, for the other implementations, RecursiveMany has a running time that is quadratic in $N$ while it is linear for

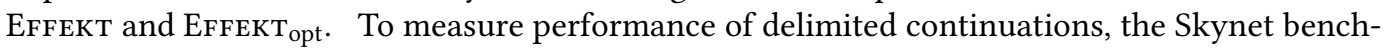
mark ${ }^{21}$ recursively spawns ten fibers until one million are created, performs some computation and aggregates the results. Each fiber corresponds to one delimited continuation. The Skynet variant never suspends, but just creates the fibers which immediately return. The SkynetSuspend variant in contrast suspends each fiber once before returning, resulting in one million continuations to be captured and resumed. Quasar and JavaFlow maintain one stack per delimited continuation / fiber each pre-allocating memory to store the function state. JavaFlow additionally maintains one stack per primitive type and copies the stack on every resumption. This leads to several million arrays copies. The Coroutines library is optimized for one shot continuations and large parts of the library are inlined in the generated bytecode. It also does not suffer from the linear stack restoration in the Skynet benchmark since the stack size of each fiber on suspension is at most one.

Performance of the Effect Library. To evaluate the performance of the overall framework, we compare EFFEKT with two other effect libraries. A monadic implementation of effect handlers in Scala "Scala Effekt"22 and the effect library "Scala Eff" ${ }^{23}$ which is based on freer monads [Kiselyov and Ishii 2015]. The results of the measurements can be found in Table 2. The CountDown8 benchmark layers eight state effects over one ambiguity effect and flips once before returning. NQueens is an effect library benchmark from the literature [Kammar et al. 2013]. The benchmarks show improvements of 2x compared to Scala Effekt and 2.5-28x compared to Scala Eff. We account the biggest performance improvement to optimizing tail resumptive operations to just be dynamic method calls. Other performance improvements compared to Scala Effekt are inlining the monadic Scala code by bytecode instrumentation and only capturing the continuation on demand (EFFEKT opt $_{\text {t }}$.

\footnotetext{
${ }^{21}$ https://github.com/atemerev/skynet

${ }^{22} \mathrm{http}: / / \mathrm{b}-$ studios.de/scala- effekt/

${ }^{23}$ http://atnos-org.github.io/eff/
} 


\section{CONCLUSIONS}

We presented the first library design for programming with effect handlers in Java. We showed how such a library can be implemented in terms of a CPS transformation and multi-prompt delimited continuations. Our CPS transformation allows trampolining, multiple resumptions and is competitive in its performance.

\section{ACKNOWLEDGMENTS}

We would like to thank Aleksandar Prokopec and Daan Leijen for inspiring discussions that helped us to improve the design and implementation of ЕғFEKT. We are also very grateful for the valuable feedback by Sam Lindley and the anonymous reviewers. This work was supported by DFG project OS 293/3-1.

\section{REFERENCES}

Andrej Bauer and Matija Pretnar. 2013. An effect system for algebraic effects and handlers. In International Conference on Algebra and Coalgebra in Computer Science. Springer, 1-16.

Andrej Bauer and Matija Pretnar. 2015. Programming with algebraic effects and handlers. fournal of Logical and Algebraic Methods in Programming 84, 1 (2015), 108-123.

Gavin Bierman, Claudio Russo, Geoffrey Mainland, Erik Meijer, and Mads Torgersen. 2012. Pause'n'Play: Formalizing Asynchronous C\#. In Proceedings of the European Conference on Object-Oriented Programming. Springer-Verlag, 233-257.

Dariusz Biernacki, Maciej Piróg, Piotr Polesiuk, and Filip Sieczkowski. 2017. Handle with Care: Relational Interpretation of Algebraic Effects and Handlers. In Proceedings of the Symposium on Principles of Programming Languages. ACM.

Jonathan Immanuel Brachthäuser and Philipp Schuster. 2017. Effekt: Extensible Algebraic Effects in Scala (Short Paper). In Proceedings of the International Symposium on Scala. ACM.

Edwin Brady. 2013. Programming and Reasoning with Algebraic Effects and Dependent Types. In Proceedings of the International Conference on Functional Programming. ACM, 133-144.

Jacques Carette, Oleg Kiselyov, and Chung-Chieh Shan. 2007. Finally Tagless, Partially Evaluated. In Proceedings of the Asian Symposium on Programming Languages and Systems. Springer LNCS 4807, 222-238.

Melvin E Conway. 1963. Design of a separable transition-diagram compiler. Commun. ACM 6, 7 (1963).

Stephen Dolan, Spiros Eliopoulos, Daniel Hillerström, Anil Madhavapeddy, KC Sivaramakrishnan, and Leo White. 2017. Concurrent system programming with effect handlers. In Proceedings of the Symposium on Trends in Functional Programming.

Stephen Dolan, Leo White, KC Sivaramakrishnan, Jeremy Yallop, and Anil Madhavapeddy. 2015. Effective concurrency through algebraic effects. In OCaml Workshop.

Iulian Dragos, Antonio Cunei, and Jan Vitek. 2007. Continuations in the Java virtual machine. In Second ECOOP Workshop on Implementation, Compilation, Optimization of Object-Oriented Languages, Programs and Systems (ICOOOLPS 2007). Technische Universität Berlin.

R Kent Dyvbig, Simon Peyton Jones, and Amr Sabry. 2007. A monadic framework for delimited continuations. Fournal of Functional Programming 17, 6 (2007), 687-730.

Michael Eichberg and Ben Hermann. 2014. A Software Product Line for Static Analyses: The OPAL Framework. In Proceedings of the 3rd ACM SIGPLAN International Workshop on the State of the Art in Java Program Analysis (SOAP '14). ACM.

Steven E. Ganz, Daniel P. Friedman, and Mitchell Wand. 1999. Trampolined Style. In Proceedings of the International Conference on Functional Programming. ACM, 18-27.

R. Hieb and R. Kent Dybvig. 1990. Continuations and Concurrency. In Proceedings of the Second ACM SIGPLAN Symposium on Principles \& Practice of Parallel Programming (PPOPP '90). ACM, 128-136.

Daniel Hillerström, Sam Lindley, Bob Atkey, and KC Sivaramakrishnan. 2017. Continuation Passing Style for Effect Handlers. In Formal Structures for Computation and Deduction (LIPIcs), Vol. 84. Schloss Dagstuhl-Leibniz-Zentrum für Informatik.

Paul Hudak. 1998. Modular Domain Specific Languages and Tools. In Proceedings of the Conference on Software Reuse. IEEE Computer Society Press, 134-142.

Ohad Kammar, Sam Lindley, and Nicolas Oury. 2013. Handlers in Action. In Proceedings of the International Conference on Functional Programming. ACM, 145-158.

Oleg Kiselyov and Hiromi Ishii. 2015. Freer Monads, More Extensible Effects. In Proceedings of the Haskell Symposium. ACM, 94-105.

Oleg Kiselyov, Amr Sabry, and Cameron Swords. 2013. Extensible Effects: An Alternative to Monad Transformers. In Proceedings of the Haskell Symposium. ACM, 59-70. 
Oleg Kiselyov and KC Sivaramakrishnan. 2016. Eff directly in OCaml. In ML Workshop.

Daan Leijen. 2014. Koka: Programming with Row Polymorphic Effect Types. In Proceedings of the Workshop on Mathematically Structured Functional Programming.

Daan Leijen. 2016. Algebraic Effects for Functional Programming. Technical Report. MSR-TR-2016-29. Microsoft Research technical report.

Daan Leijen. 2017a. Implementing Algebraic Effects in C. In Proceedings of the Asian Symposium on Programming Languages and Systems. Springer International Publishing, Cham, Switzerland, 339-363.

Daan Leijen. 2017b. Structured Asynchrony with Algebraic Effects. In Proceedings of the Workshop on Type-Driven Development. ACM, 16-29.

Daan Leijen. 2017c. Type directed compilation of row-typed algebraic effects. In Proceedings of the Symposium on Principles of Programming Languages. 486-499.

Sam Lindley. 2018. Encapsulating effects, In Algebraic Effect Handlers go Mainstream (Dagstuhl Seminar 18172). Dagstuhl Reports 8, 4 .

Sam Lindley, Conor McBride, and Craig McLaughlin. 2017. Do Be Do Be Do. In Proceedings of the Symposium on Principles of Programming Languages. ACM, 500-514.

Florian Loitsch. 2007. Exceptional continuations in JavaScript. In Workshop on Scheme and Functional Programming.

Bruno C. d. S. Oliveira and William R. Cook. 2012. Extensibility for the Masses: Practical Extensibility with Object Algebras. In Proceedings of the European Conference on Object-Oriented Programming. Springer LNCS 7313, 2-27.

Greg Pettyjohn, John Clements, Joe Marshall, Shriram Krishnamurthi, and Matthias Felleisen. 2005. Continuations from Generalized Stack Inspection. In Proceedings of the International Conference on Functional Programming. ACM, 216-227.

Gordon Plotkin and John Power. 2003. Algebraic operations and generic effects. Applied Categorical Structures 11, 1 (2003), 69-94.

Gordon Plotkin and Matija Pretnar. 2009. Handlers of algebraic effects. In European Symposium on Programming. SpringerVerlag, 80-94.

Joe Gibbs Politz, Alejandro Martinez, Matthew Milano, Sumner Warren, Daniel Patterson, Junsong Li, Anand Chitipothu, and Shriram Krishnamurthi. 2013. Python: The Full Monty. In Proceedings of the Conference on Object-Oriented Programming, Systems, Languages and Applications. ACM, 217-232.

Ron Pressler. 2017. Loom Project: Fibers and Continuations for the fava Virtual Machine. OpenJDK Project. HotSpot Group. http://mail.openjdk.java.net/pipermail/discuss/2017-September/004390.html

Aleksandar Prokopec and Fengyun Liu. 2018. Theory and practice of coroutines with snapshots. In Proceedings of the European Conference on Object-Oriented Programming. Schloss Dagstuhl-Leibniz-Zentrum für Informatik.

John C. Reynolds. 1972. Definitional Interpreters for Higher-Order Programming Languages. In Proceedings of the ACM annual conference. ACM, 717-740.

Tatsurou Sekiguchi, Takahiro Sakamoto, and Akinori Yonezawa. 2001. Advances in Exception Handling Techniques. Springer-Verlag, Heidelberg, Berlin, Germany, Chapter Portable Implementation of Continuation Operators in Imperative Languages by Exception Handling, 217-233.

Lukas Stadler, Christian Wimmer, Thomas Würthinger, Hanspeter Mössenböck, and John Rose. 2009. Lazy continuations for Java virtual machines. In Proceedings of the International Conference on Principles and Practice of Programming in fava. ACM, 143-152.

Nicolas Wu and Tom Schrijvers. 2015. Fusion for Free - Efficient Algebraic Effect Handlers. In Proceedings of the Conference on Mathematics of Program Construction. Springer LNCS 9129. 